\title{
Factores de riesgo asociados a complicaciones cardiovasculares en pacientes con enfermedad de Kawasaki en el noroeste de México
}

\author{
Olivia A. Flores-Montes ${ }^{1}$, Jaime Valle-Leal' ${ }^{*}$, Roberto Arreguin-Reyes ${ }^{2}$ y Jesús M. Armenta-Velderrain ${ }^{1}$ \\ ${ }^{1}$ Departamento de Pediatría; ${ }^{2}$ Departamento de Reumatología. Hospital General Regional 1, Instituto Mexicano del Seguro Social, Ciudad Obregón, \\ Sonora, México
}

\begin{abstract}
Resumen
Introducción: La enfermedad de Kawasaki (EK) es una vasculitis sistémica aguda de pequeños y medianos vasos, que se presenta principalmente en niños. Se manifiesta como síndrome febril aunado a datos de vasculitis, y puede causar anormalidades en las arterias coronarias en el $25 \%$ de los pacientes no tratados. El objetivo de este estudio fue describir el comportamiento clínico y la identificación de factores de riesgo para complicaciones cardiovasculares en pacientes pediátricos con EK atendidos en un hospital de segundo nivel del noroeste de México. Métodos: Bajo un diseño de serie de casos, se estudiaron pacientes pediátricos con diagnóstico de EK. Se midieron variables clínicas, de laboratorio y presencia de complicaciones cardiacas, y se estimó la probabilidad de riesgo con razón de momios (RM) y asociación con prueba de ji al cuadrado. Resultados: Se incluyeron 12 pacientes y predominó el sexo femenino; la edad media de presentación de la EK fue de 2 años. La presentación clínica fue completa en el 100\% de los casos y hubo además manifestaciones atípicas. El 50\% de los pacientes estudiados presentaron complicaciones cardiovasculares; la más común fue afección coronaria (33\%). Las variables sexo masculino, edad menor de 2 años y anemia tuvieron RM de 5.5 y 10 de presentar complicaciones cardiovasculares. Conclusiones: Las complicaciones cardiovasculares de la EK son frecuentes (más del $30 \%$ de los pacientes). Las variables sexo masculino, edad menor de 2 años y anemia incrementaron la probabilidad de riesgo para la presencia de complicaciones cardiovasculares.
\end{abstract}

Palabras clave: Enfermedad de Kawasaki. Comportamiento clínico. Aneurismas. Miocarditis. Inmunoglobulina.

\section{Risk factors related to cardiovascular complications in patients diagnosed with Kawasaki disease in northwestern Mexico}

\begin{abstract}
Background: Kawasaki disease (KD) is an acute systemic vasculitis of small and medium vessels, which occurs primarily in children; it manifests itself as a febrile syndrome coupled with vasculitis data and can cause coronary artery abnormalities in $25 \%$ of untreated patients. The objective of this study was to describe the clinical behavior and to identify risk factors for cardiovascular complications in pediatric patients with KD, in a second level hospital in Northwestern Mexico. Methods: Under
\end{abstract}

\section{Correspondencia:}

*Jaime Valle Leal

E-mail: valle_jaime1@ @otmail.com
Disponible en internet: 14-05-2018 Bol Med Hosp Infant Mex. 2018;75:145-152 www.bmhim.com

1665-1146/C 2018. Hospital Infantil de México Federico Gómez, impreso por Permanyer México SA de CV, todos los derechos reservados. 
a case series design, we studied pediatric patients with diagnosis of KD. We measured clinical variables, laboratory values and the presence of cardiac complications; the probability of risk was determined with odds ratio $(O R)$ and the association with chi squared test. Results: 12 patients were included, and the female gender predominated; the mean age of presentation of KD was 2 years. The clinical presentation was complete in $100 \%$ of the cases and patients also presented atypical manifestations. $50 \%$ of the patients studied had cardiovascular complications, the most common of which was coronary disease (33\%). The variables male gender, age under 2 years and anemia reported OR of 5.5 and 10 to present cardiovascular complications. Conclusions: Cardiovascular complications of KD are frequent (more than $30 \%$ ). Male gender, age under 2 years and anemia increase the probability of risk for the presence of cardiovascular complications.

Key words: Kawasaki disease. Clinical behavior. Aneurysms. Myocarditis. Immunoglobulin.

\section{Introducción}

La enfermedad de Kawasaki (EK) es una vasculitis sistémica aguda de pequeños y medianos vasos que se presenta principalmente en niños. Se manifiesta como síndrome febril aunado a datos de vasculitis, y puede causar anormalidades en las arterias coronarias en el $25 \%$ de los pacientes no tratados ${ }^{1-3}$. Su incidencia es mayor en los menores de 5 años del sexo masculino, y el mayor número de casos se presenta en las estaciones de invierno y primavera ${ }^{1-5}$.

La incidencia de la enfermedad no es bien conocida. En Japón se han reportado 218 casos por cada 100,000 menores de 5 años ${ }^{6}$; en los EE.UU., 20 casos anuales por cada 100,000; y en algunos países latinoamericanos, 11 casos anuales por cada 100,000 menores de 5 años. En México no se conoce la incidencia real, pues solo existe reporte de algunas series de casos de la enfermedad ${ }^{4,7-11}$.

La etiología no está claramente definida, pero se cree que existen factores desencadenantes en individuos genéticamente susceptibles que precipitan la cascada de eventos inflamatorios que dan lugar a la enfermedad ${ }^{12-16}$.

La ausencia de pruebas de laboratorio especificas hace que el diagnóstico sea de tipo clínico y esté basado en los criterios propuestos por el Dr. Kawasaki, que fueron confirmados posteriormente por la American Heart Association (AHA $)^{5}$. Existen, además, criterios de laboratorio suplementarios, los cuales no son específicos, pero son útiles cuando el paciente presenta características clínicas que son sugestivas, pero no diagnósticas, de la enfermedad (incompletos) 5,17.

Durante la fase aguda de la enfermedad existen infiltración e inflamación de células cardiacas que pueden generar afección coronaria, miocárdica, valvular y pericárdica; estas alteraciones pueden estar presentes en más del $40 \%$ de los pacientes ${ }^{2}$, por lo que deben ser buscadas intencionalmente mediante ecocardiograma bidimensional, el cual debe realizarse en el momento del diagnóstico de la enfermedad ${ }^{18}$.
Se han identificado algunos predictores de enfermedad coronaria, como por ejemplo la presentación incompleta de la enfermedad, el sexo masculino, la edad menor de 1 año o mayor de 5 años, y fiebre de 6 días o más de duración. El puntaje de Harada identifica a los pacientes con EK y alto riesgo de desarrollo de aneurismas coronarios; esta clasificación considera riesgo cuando el puntaje es $\geq 4^{2,4,5}$.

La baja sospecha clínica de la enfermedad sigue retardando el diagnóstico y, con ello, el aumento en el riesgo de complicaciones cardiovasculares ${ }^{19}$. Es importante conocer cómo se expresa la enfermedad en las diferentes edades y en las distintas regiones del país. El objetivo de este estudio fue describir el comportamiento clínico y la identificación de factores de riesgo para complicaciones cardiovasculares en pacientes pediátricos con EK, atendidos en un hospital de segundo nivel del noroeste de México.

\section{Métodos}

Previa autorización por el comité de ética e investigación local, se realizó un estudio de casos con pacientes pediátricos con diagnóstico de EK, atendidos en un hospital de segundo nivel de atención en el noroeste de México, durante el periodo comprendido entre enero de 2013 y julio de 2016. Las variables estudiadas fueron las siguientes: complicaciones cardiovasculares (presentes o ausentes y tipo de afección), comportamiento clínico (presentación completa, incompleta, manifestaciones atípicas), puntaje de Harada, sexo, edad, cuenta de leucocitos, hemoglobina, volumen corpuscular medio, cuenta plaquetaria, proteína $C$ reactiva $(P C R)$, velocidad de sedimentación globular (VSG), valores séricos de albúmina, eritrocituria, proteinuria, piuria, cilindruria, alanino aminotransferasa (ALT), aspartato aminotransferasa (AST), urea, creatinina, estado nutricional (eutrofia, sobrepeso, obesidad, obesidad grave), estación del año en la cual se diagnosticó la enfermedad (primavera, verano, otoño o 
invierno), tratamiento (adecuado o inadecuado) y proteinuria (positiva o negativa). Se consideró leucocitosis una cuenta celular mayor de $15,000 / \mathrm{mm}^{3}$; plaquetosis, una cuenta plaquetaria mayor de $450,000 / \mathrm{mm}^{3}$; anemia, cifras de hemoglobina $(\mathrm{Hb})$ menores de $10 \mathrm{~g} / \mathrm{dl}$; hipoalbuminemia, concentraciones de albúmina menores de $3 \mathrm{~g} / \mathrm{dl}$; PCR positiva, valores mayores de $1 \mathrm{mg} /$ dl; VSG elevada, valores séricos mayores de $40 \mathrm{~mm} / \mathrm{h}$; eritrocituria, presencia de cinco o más eritrocitos por campo en el sedimento urinario; leucocituria, presencia de más de cinco leucocitos por campo en sedimento urinario; y ALT y AST elevadas, valores séricos mayores de $40 \mathrm{U} / \mathrm{l}$.

Para definir el comportamiento clínico, se clasificó de acuerdo con los criterios clínicos de presentación de la enfermedad como fiebre persistente de más de 5 días de evolución y al menos cuatro de los siguientes criterios: 1) cambios en las extremidades; 2) exantema polimorfo; 3) inyección conjuntival bulbar bilateral sin exudado; 4) cambios en los labios y la cavidad oral; y 5) linfadenopatía aguda no purulenta, clasificándose como EK completa. En cambio, si se cumplen menos de cuatro criterios y con alteraciones coronarias detectadas en el ecocardiograma se considera EK incompleta. Si los pacientes presentaban alteraciones renales (proteinuria, eritrocituria, cilindruria, leucocituria, piuria estéril, elevación de compuestos nitrogenados), gastrointestinales (diarrea, vómito, dolor abdominal), hepáticas (hiperbilirrubinemia, hipertransaminasemia, hipoalbuminemia) o neurológicas (irritabilidad extrema, meningitis aséptica, pérdida neurosensorial), estas se clasificaron como manifestaciones atípicas o no comunes.

Se valoraron, además, el tiempo de duración de la fiebre (en días); las complicaciones cardiovasculares integradas por ecocardiograma (dilatación coronaria con un diámetro interno mayor de $2.5 \mathrm{~mm}$, aneurisma coronario pequeño si midió menos de $5 \mathrm{~mm}$, mediano entre 5 y $8 \mathrm{~mm}$ y gigante si midió más de $8 \mathrm{~mm}$ ); el tratamiento recibido y el tiempo de evolución de la enfermedad en que se administró, así como el puntaje de Harada de cada uno de los pacientes para calcular el riesgo de desarrollo de aneurismas coronarios.

De acuerdo con el tratamiento, se consideró la administración de salicilatos, glucocorticoides e inmunoglobulina, dividiéndose esta última en tres grupos: 1) administración temprana, aquellos que recibieron $2 \mathrm{~g} / \mathrm{kg}$ en infusión continua de $12 \mathrm{~h}$ antes de los 10 días de evolución; 2) administración tardía, quienes recibieron $2 \mathrm{~g} / \mathrm{kg}$ después de los 10 días de evolución; y 3 ) los que no recibieron inmunoglobulina o en quienes la dosis fue fraccionada.
Los datos recolectados se transfirieron a una base electrónica y se analizaron mediante el paquete estadístico SPSS versión 2.0, en español. Se utilizó estadística descriptiva. Las variables cualitativas se describieron en medidas de frecuencia (porcentajes), medianas y rangos intercuartílicos para variables cuantitativas, y determinación de la razón de momios (RM) para determinar la probabilidad de riesgo. La asociación entre variables cualitativas se determinó mediante la prueba de ji al cuadrado.

\section{Resultados}

Se estudiaron 12 pacientes, 8 de sexo femenino y 4 de sexo masculino (relación 2:1), cuya mediana de edad fue de 2.05 años; 4 (33\%) pacientes eran menores de 2 años y 8 (67\%) tenían edades comprendidas entre 2 y 3.5 años (Tabla 1).

Con respecto a la fase de la enfermedad al momento del diagnóstico, se detectaron 8 pacientes $(67 \%)$ en fase aguda y $4(33 \%)$ en fase subaguda. La duración de la fiebre fue variable, con una mediana de 9.5 días. La fiebre duró 10 o más días en $6(50 \%)$ de los casos $^{6}$ (Tabla 1).

El comportamiento clínico al momento del diagnóstico fue de EK completa en el $100 \%$ de los casos; asimismo, todos presentaron manifestaciones atípicas (también llamadas no comunes) (Tabla 1). En la tabla 2 se describen las manifestaciones clínicas típicas y atípicas encontradas en este grupo de pacientes.

Las alteraciones de laboratorio encontradas con mayor frecuencia fueron leucocitosis en el $75 \%$ de los pacientes, trombocitosis en el $67 \%$, hipoalbuminemia y AST elevada en el $50 \%$ de los pacientes. En cuanto al análisis del sedimento urinario, el $33.3 \%$ de los pacientes presentó cilindros hialinos, el 25\% proteinuria, piuria y eritrocituria, y el $16.6 \%$ cilindros granulosos.

Acerca del tratamiento, a 10 pacientes $(83 \%)$ se les administró un tratamiento adecuado; a tres de ellos se les administró una segunda dosis de inmunoglobulina por persistencia de la fiebre. A los 12 pacientes se les administraron salicilatos, y solo uno recibió glucocorticoides.

En $6(50 \%)$ de los pacientes se encontraron complicaciones cardiovasculares, 3 (3/8) de ellos del sexo femenino y 3 (3/4) del sexo masculino. En la tabla 3 se describen con detalle las características de este grupo de pacientes.

De los pacientes con complicaciones cardiovasculares, en $4(67 \%)$ se encontró afección coronaria: dos presentaron dilatación de la coronaria izquierda y uno 
Tabla 1. Descripción general de pacientes pediátricos $(n=12)$ con enfermedad de Kawasaki atendidos en un hospital de segundo nivel de atención en el noroeste de México

\begin{tabular}{|c|c|c|}
\hline \multicolumn{2}{|l|}{ Variable } & \multirow{2}{*}{$\begin{array}{c}\text { Frecuencia/porcentaje } \\
\qquad \begin{array}{c}8 / 12(67 \%) \\
4 / 12(33 \%)\end{array}\end{array}$} \\
\hline Sexo & $\begin{array}{l}\text { Femenino } \\
\text { Masculino }\end{array}$ & \\
\hline Edad & $\begin{array}{l}<2 \text { años } \\
\geq 2 \text { años }\end{array}$ & $\begin{array}{l}4 / 12(33 \%) \\
8 / 12(67 \%)\end{array}$ \\
\hline Estado nutricional & $\begin{array}{l}\text { Eutrófico } \\
\text { Sobrepeso y obesidad }\end{array}$ & $\begin{array}{l}5 / 12(42 \%) \\
7 / 12(58 \%)\end{array}$ \\
\hline \multirow[t]{2}{*}{ Estación del año } & \multirow{2}{*}{$\begin{array}{l}\text { Primavera } \\
\text { Verano } \\
\text { Otoño } \\
\text { Invierno }\end{array}$} & $\begin{array}{c}4 / 12(34 \%) \\
1 / 12(8 \%)\end{array}$ \\
\hline & & $\begin{array}{l}1 / 12(8 \%) \\
6 / 12(50 \%)\end{array}$ \\
\hline Fase de evolución & $\begin{array}{l}\text { Aguda } \\
\text { Subaguda }\end{array}$ & $\begin{array}{l}8 / 12(66.6 \%) \\
4 / 12(33.3 \%)\end{array}$ \\
\hline Comportamiento clínico & $\begin{array}{l}\text { Completo } \\
\text { Incompleto }\end{array}$ & $\begin{array}{c}12 / 12(100 \%) \\
0 / 12(0 \%)\end{array}$ \\
\hline Duración de la fiebre & $\begin{array}{l}<10 \text { días } \\
\geq 10 \text { días }\end{array}$ & $\begin{array}{l}6(50 \%) \\
6(50 \%)\end{array}$ \\
\hline Manifestaciones extramucocutáneas & $\begin{array}{l}\text { Presentes } \\
\text { Ausentes }\end{array}$ & $\begin{array}{l}12 / 12(100 \%) \\
0 / 12(0 \%)\end{array}$ \\
\hline Tratamiento con inmunoglobulina intravenosa & $\begin{array}{l}\text { Adecuado } \\
\text { Inadecuado }\end{array}$ & $\begin{array}{l}10 / 12(83 \%) \\
2 / 12(17 \%)\end{array}$ \\
\hline Complicaciones cardiovasculares & $\begin{array}{l}\text { Lesión coronaria izquierda } \\
\text { Lesión coronaria derecha } \\
\text { Aneurisma de coronaria } \\
\text { Hipertrofia ventrículo izquierdo }\end{array}$ & $\begin{array}{l}2 / 12(17 \%) \\
1 / 12(18 \%) \\
1 / 12(18 \%) \\
2 / 12(17 \%)\end{array}$ \\
\hline
\end{tabular}

de la coronaria derecha, y uno presentó aneurisma en ambas coronarias, de tamaño mediano.

En la tabla 4 se presenta la asociación de algunas alteraciones de laboratorio con la presencia de complicaciones cardiovasculares; destacan la eritrocituria y la anemia, con una probabilidad $(p)$ de 0.09 y 0.12 , respectivamente. Se calculó la probabilidad de riesgo para complicaciones cardiovasculares y se determinó una RM de 10 para anemia; para edad menor de 2 años, sexo masculino y presencia de cilindros hialinos se determinó una RM de 5 (Tabla 4).

En la tabla 5 se describen las medianas y los rangos intercuartílicos para la edad, los días de fiebre y los resultados de laboratorio en pacientes con y sin complicaciones cardiovasculares; destaca la mediana de $\mathrm{Hb}$, de 9.5, en los pacientes con complicaciones contra 11.25 en los pacientes sin complicación.

\section{Discusión}

En este estudio se encontró predominio del sexo femenino, con una relación masculino:femenino de 1:2, distinto a lo reportado por Sotelo-Cruz², quien encontró un predominio del sexo masculino en proporción 3.3:1. Los casos fueron más frecuentes en menores de 2 años, distinto a lo reportado por series nacionales en las que predominaron los pacientes entre 3 y 5 años de edad $^{2,3,20}$.

De acuerdo con la presentación estacional, la mitad de los casos tuvieron lugar en invierno y solo un tercio en primavera, lo cual difiere de lo reportado en los estudios nacionales, en los que se determinó una mayor frecuencia de casos en primavera ${ }^{3}$.

La mayoría de los pacientes se detectaron durante la fase aguda, con un promedio de fiebre de 9.5 días, siendo similar a lo reportado por Morales-Quispe, et al. ${ }^{20}$, encontrándose muy cercano al límite de lo recomendado por la AHA para disminuir el riesgo de complicaciones coronarias ${ }^{7}$.

En cuanto al comportamiento clínico, todos los pacientes cumplieron con los criterios para definir EK completa, similar a lo encontrado en las series más grandes publicadas en México ${ }^{2,6,11}$. Asimismo, todos los pacientes presentaron manifestaciones atípicas (no 
Tabla 2. Manifestaciones típicas y atípicas (no comunes) en pacientes pediátricos $(\mathrm{N}=12)$ con enfermedad de Kawasaki atendidos en un hospital de segundo nivel de atención en el noroeste de México

\begin{tabular}{|c|c|c|}
\hline \multirow[t]{2}{*}{ Manifestaciones típicas } & \multicolumn{2}{|c|}{ Frecuencia } \\
\hline & $\mathbf{N}$ & $(\%)$ \\
\hline Fiebre $>10$ días & $8 / 12$ & $(67 \%)$ \\
\hline Descamación & $9 / 12$ & $(75 \%)$ \\
\hline Exantema & $11 / 12$ & (92\%) \\
\hline Hiperemia conjuntival & $10 / 12$ & $(83 \%)$ \\
\hline Hiperemia oral & $10 / 12$ & $83 \%)$ \\
\hline Adenopatías & $9 / 12$ & $(75 \%)$ \\
\hline Hemorragia oral & $6 / 12$ & $(50 \%)$ \\
\hline Manifestaciones atípicas & & \\
\hline $\begin{array}{l}\text { Respiratorias } \\
\text { Rinorrea } \\
\text { Tos } \\
\text { Dificultad respiratoria } \\
\text { Derrame Pleural }\end{array}$ & $\begin{array}{l}6 / 12 \\
6 / 12 \\
2 / 12 \\
1 / 12\end{array}$ & $\begin{array}{c}(50 \%) \\
(50 \%) \\
(17 \%) \\
(8 \%)\end{array}$ \\
\hline $\begin{array}{l}\text { Gastrointestinales } \\
\text { Dolor abdominal } \\
\text { Diarrea } \\
\text { Sangrado tubo digestivo }\end{array}$ & $\begin{array}{l}7 / 12 \\
5 / 12 \\
3 / 12\end{array}$ & $\begin{array}{l}(58 \%) \\
(42 \%) \\
(25 \%)\end{array}$ \\
\hline $\begin{array}{l}\text { Renales } \\
\text { Hematuria } \\
\text { Proteinuria } \\
\text { Lesión renal aguda }\end{array}$ & $\begin{array}{l}3 / 12 \\
3 / 12 \\
2 / 12\end{array}$ & $\begin{array}{l}(25 \%) \\
(25 \%) \\
(17 \%)\end{array}$ \\
\hline $\begin{array}{l}\text { Articulares } \\
\text { Artritis } \\
\text { Artralgias }\end{array}$ & $\begin{array}{l}3 / 12 \\
5 / 12\end{array}$ & $\begin{array}{l}(25 \%) \\
(42 \%)\end{array}$ \\
\hline $\begin{array}{l}\text { Cardiovasculares } \\
\text { Miocarditis } \\
\text { Hipertensión arterial }\end{array}$ & $\begin{array}{l}2 / 12 \\
1 / 12\end{array}$ & $\begin{array}{c}(17 \%) \\
(8 \%)\end{array}$ \\
\hline $\begin{array}{l}\text { Hepáticas } \\
\text { Hepatomegalia } \\
\text { Hipertransaminasemia } \\
\text { Hiperbilirrubinemia } \\
\text { Hidrops de vesícula biliar }\end{array}$ & $\begin{array}{l}3 / 12 \\
5 / 12 \\
3 / 12 \\
1 / 12\end{array}$ & $\begin{array}{l}(25 \%) \\
(42 \%) \\
(25 \%) \\
(8 \%)\end{array}$ \\
\hline
\end{tabular}

comunes), proporción mayor que la reportada en otras series $^{2,3,6,9,20,21}$. Los síntomas atípicos (no comunes) más frecuentes fueron los gastrointestinales (dolor $a b-$ dominal y diarrea), respiratorios (tos) y articulares (artralgias), similar a lo encontrado por Sotelo-Cruz ${ }^{2}$, quien halló un predominio de dolor articular, hepatomegalia, tos, dolor abdominal, náuseas y vómito.

Las alteraciones de laboratorio más frecuentes fueron leucocitosis, trombocitosis, hipoalbuminemia y elevación de AST, que se han observado en la mayoría de los estudios publicados. La PCR resultó elevada en todos los pacientes, pero en todos los casos fue menor de $3 \mathrm{mg} / \mathrm{dl}$.
Tabla 3. Descripción de pacientes pediátricos con enfermedad de Kawasaki y complicaciones cardiovasculares ( $N=6$ ) de un hospital de segundo nivel de atención en el noroeste de México

\begin{tabular}{|l|l|l|}
\hline Variable & Frecuencia \\
\hline Sexo & $\begin{array}{l}\text { Masculino } \\
\text { Femenino }\end{array}$ \\
\hline Edad & $\begin{array}{l}<\text { años } \\
\geq 2 \text { años }\end{array}$ & $3 / 6(50 \%)$ \\
\hline Estado nutricional & Eutrofia & $3 / 6(50 \%)$ \\
\hline Manifestaciones & Sobrepeso y obesidad & $3 / 6(50 \%)$ \\
\hline atípicas & Presentes & $2 / 6(33 \%)$ \\
\hline Puntaje de Harada & Positivo & $6 / 6(100 \%)$ \\
\hline Duración de la fiebre & $<10$ días & $1 / 6(17 \%)$ \\
\hline Tratamiento & $>10$ días & $3 / 6(50 \%)$ \\
\hline & Adecuado & $3 / 6(50 \%)$ \\
\hline
\end{tabular}

Todos los pacientes recibieron inmunoglobulina intravenosa $(2 \mathrm{~g} / \mathrm{kg})$. La mayoría de ellos recibió un tratamiento adecuado con inmunoglobulina temprana y salicilatos; solo dos pacientes recibieron inmunoglobulina de forma tardía y un paciente requirió glucocorticoide por persistencia de la fiebre. Estos tres pacientes presentaron alteraciones coronarias, lo cual se relaciona con lo reportado por Garrido-García, et al. ${ }^{6}$ en su serie. Los dos pacientes que recibieron inmunoglobulina intravenosa después del día 10 de evolución y el que recibió glucocorticoides presentaron alteraciones coronarias; dos de ellos mostraron dilatación de coronarias, y uno tuvo aneurisma de ambas coronarias. De ellos, solo el que recibió glucocorticoides presentó un puntaje de Harada positivo. El $50 \%$ de los pacientes en esta serie presentaron alteraciones ecocardiografías, porcentaje superior al 32\% reportado por SoteIo-Cruz ${ }^{2}$, el 29.4\% reportado por García-Rodríguez, et al. ${ }^{11}$ y el $35 \%$ reportado por Surjit, et al..$^{22}$; y similar al $54 \%$ reportado por Gil-Veloz, et al. ${ }^{3}$ en su serie. Nuestros resultados pueden estar influenciados por las características de los pacientes de nuestra serie, pues predomina el sexo femenino, la mediana de edad es de 2.1 años (mucho menor que la reportada en otras series $^{2,3,20}$ ) y hay una alta frecuencia de manifestaciones atípicas (no comunes).

De los pacientes con alteraciones ecocardiográficas, el $50 \%$ pertenecían al sexo femenino y el otro $50 \%$ al sexo masculino; sin embargo, respecto al número total de pacientes de cada sexo, se observan más complicaciones cardiovasculares en los niños (75\%) que en 
Tabla 4. Asociación de alteración de parámetros bioquímicos y estimación de riesgo para complicaciones cardiovasculares en pacientes pediátricos con enfermedad de Kawasaki $(\mathrm{N}=12)$ atendidos en un hospital de segundo nivel de atención en el noroeste de México

\begin{tabular}{|c|c|c|c|c|c|}
\hline & \multirow{2}{*}{$\begin{array}{c}\text { Con complicación } \\
\text { cardiovascular }\end{array}$} & \multirow{2}{*}{$\begin{array}{c}\text { Sin } \\
\text { complicación } \\
\text { cardiovascular }\end{array}$} & & & \\
\hline & & & $\mathbf{p}$ & $\mathbf{R M}$ & IC $95 \%$ \\
\hline VSG $>40 \mathrm{~mm} / \mathrm{h}$ & $4 / 9(44 \%)$ & $5 / 9(56 \%)$ & 0.50 & 0.6 & $(0.2-1.9)$ \\
\hline PCR $>1 \mathrm{mg} / \mathrm{dl}$ & $4 / 7(57 \%)$ & $3 / 7(43 \%)$ & 0.28 & 2 & $(0.56-7)$ \\
\hline Anemia & $5 / 7(71 \%)$ & $2 / 7(29 \%)$ & 0.12 & 10 & (0.6-154 \\
\hline Leucocitosis & $2 / 6(67 \%)$ & $1 / 6(33 \%)$ & 0.50 & 1.5 & $(0.5-4.4)$ \\
\hline Trombocitosis & $4 / 6(67 \%)$ & $4 / 6(67 \%)$ & 1 & 1 & $(0.3-3.3)$ \\
\hline Urea elevada & $2 / 3(57 \%)$ & $1 / 3(33 \%)$ & 0.50 & 2.5 & (0.1-38) \\
\hline Creatinina elevada & $3 / 5(60 \%)$ & $2 / 5(40 \%)$ & 0.50 & 2 & $(0.1-20)$ \\
\hline Hipoalbuminemia & $4 / 6(67 \%)$ & $2 / 6(33 \%)$ & 0.28 & 4 & $(0.3-44)$ \\
\hline ALT elevada & $3 / 5(60 \%)$ & $2 / 5(40 \%)$ & 0.55 & 2 & $(0.2-20)$ \\
\hline AST elevada & $3 / 6(50 \%)$ & $3 / 6(50 \%)$ & 1 & 1 & $(0.1-9.6)$ \\
\hline Hiperbilirrubinemia & $2 / 3(67 \%)$ & $1 / 3(33 \%)$ & 0.50 & 2.5 & $(0.1-38)$ \\
\hline Proteinuria & $2 / 6(67 \%)$ & $1 / 6(33 \%)$ & 0.13 & 1.5 & $(0.5-4.4)$ \\
\hline Eritrocituria & $3 / 3(100 \%)$ & $0(0 \%)$ & 0.09 & - & - \\
\hline Cilindros hialinos & $3 / 4(75 \%)$ & $1 / 4(25 \%)$ & 0.27 & 5 & $(0.3-72)$ \\
\hline Cilindros granulosos & $2 / 2(100 \%)$ & $0(0 \%)$ & 0.22 & - & - \\
\hline Leucocituria & $2 / 3(67 \%)$ & $1 / 3(33 \%)$ & 0.50 & 2.5 & $(0.1-38)$ \\
\hline Fiebre $>10$ días & $3 / 6(50 \%)$ & $1 / 6(17 \%)$ & 0.22 & 2 & $(0.6-5.7)$ \\
\hline Sobrepeso y obesidad & $4 / 6(67 \%)$ & $2 / 6(33 \%)$ & 0.34 & 1.4 & $(0.4-4.9)$ \\
\hline Sexo masculino & $3 / 4(75 \%)$ & $1 / 4(25 \%)$ & 0.27 & 5 & $(0.7-72)$ \\
\hline Sexo femenino & $3 / 8(38 \%)$ & $5 / 8(62 \%)$ & 0.27 & 0.2 & $(0.01-2)$ \\
\hline Edad $<2$ años & $3 / 4(75 \%)$ & $1 / 4(25 \%)$ & 0.27 & 5 & $(0.7-72)$ \\
\hline Edad $\geq 2$ años & $3 / 8(38 \%)$ & $5 / 9(62 \%)$ & 0.27 & 0.2 & $(0.01-2)$ \\
\hline
\end{tabular}

ALT: alanino aminotransferasa; AST: aspartato aminotransferasa; IC: intervalo de confianza; p: probabilidad calculada por prueba exacta de Fisher; PCR: proteína C reactiva; RM: razón de momios; VSG: velocidad de sedimentación globular.

las niñas (38\%). El $50 \%$ de los pacientes con complicaciones cardiovasculares eran menores de 2 años; el otro $50 \%$ tenía entre 2 y 3.5 años de edad.

Dentro de los parámetros de laboratorio, se encontró una probabilidad de riesgo 10 veces mayor de desarrollar complicaciones cardiovasculares en los pacientes que presentaban anemia, y cinco veces mayor en los pacientes de sexo masculino, con cilindros hialinos en el sedimento urinario y en aquellos menores de 2 años. La elevación de la PCR y de la VSG, una cuenta de leucocitos mayor de 12,000, valores bajos de $\mathrm{Hb}$, el hematocrito menor del 35\%, la albumina sérica disminuida, la elevación de las transaminasas y la hiponatremia se han reportado en otras publicaciones como factores de riesgo para presentar complicaciones cardiovasculares ${ }^{23}$.

La mediana de $\mathrm{Hb}$ en los pacientes con complicación cardiovascular fue de 9.6, contra 11.25 en los pacientes sin complicaciones; resultados similares a los reportados por Caballero-Mora, et al. ${ }^{23}$, quienes reportan una media de $\mathrm{Hb}$ de 10.3 en pacientes con complicaciones y de 11.42 en pacientes sin 
Tabla 5. Descripción de las medianas y los rangos intercuartílicos de las características clínicas y de laboratorio de pacientes pediátricos con enfermedad de Kawasaki con y sin complicaciones cardiovasculares $(N=12)$

\begin{tabular}{|l|c|c|}
\hline & Con complicación cardiovascular & Sin complicación cardiovascular \\
\cline { 2 - 3 } & Mediana (RI) & Mediana (RI) \\
\hline Edad (años) & $1.7(1.9)$ & $2.15(0.9)$ \\
\hline Fiebre (días de duración) & $12(9)$ & $8(3)$ \\
\hline VSG (mm/h) & $46(8)$ & $47.5(5)$ \\
\hline Proteína C reactiva (mg/dl) & $1.25(0.5)$ & $1.05(0.3)$ \\
\hline Hemoglobina (g/l) & $9.6(0.8)$ & $11.25(1.6)$ \\
\hline Volumen corpuscular medio (fl) & $82(9)$ & $90(5)$ \\
\hline Leucocitos (leucocitos/mm3) & $12,700(6180)$ & $10,100(2700)$ \\
\hline Plaquetas (plaquetas/mm3) & $495,500(341,000)$ & $415,000(174,000)$ \\
\hline Urea (mg/dl) & $20.5(22)$ & $22(18)$ \\
\hline Creatinina (mg/dl) & $0.65(0.5)$ & $0.45(0.5)$ \\
\hline Albúmina g/dl & $2.7(0.9)$ & $3.3(0.7)$ \\
\hline ALT (U/l) & $52.5(30)$ & $40.5(22)$ \\
\hline AST (U/l) & $47.5(53)$ & $40(9)$ \\
\hline Bilirrubina total (mg/dl) & $0.95(0.7)$ & $0.4(0.4)$ \\
\hline
\end{tabular}

ALT: alanino aminotransferasa; AST: aspartato aminotransferasa; RI: rango intercuartílico; VSG: velocidad de sedimentación globular.

complicaciones. La mediana de días de fiebre fue de 12 para los pacientes con complicaciones cardiovasculares, contra 8 días en los pacientes sin complicaciones cardiovasculares.

La detección temprana es muy importante para evitar complicaciones cardiovasculares. En esta serie, la mayoría de los pacientes tuvieron un tratamiento adecuado y las complicaciones cardiovasculares se presentaron en la mitad de los casos; el 33\% correspondía a afección coronaria, similar a lo reportado en otras series nacionales ${ }^{3,6}$. En Japón se ha observado una disminución de las secuelas cardiovasculares (aneurismas coronarios e infartos al miocardio) gracias a la detección de la enfermedad en fase aguda y a su manejo adecuado ${ }^{9}$. Según lo reportado por Gil-Veloz, et al. ${ }^{3}$ y por Morales-Quispe, et al. ${ }^{20}$, la administración de gammaglobulina en los primeros días de la enfermedad disminuye el riesgo de complicaciones cardiovasculares, en especial de aneurismas residuales.

Si bien no se ha reportado el riesgo de la eritrocituria para el desarrollo de complicaciones cardiovasculares, como se encontró en el presente estudio, se debería buscar la asociación de este signo $u$ otros en series más grandes de pacientes con el fin de mejorar la predicción del riesgo de complicaciones y tratarlas a tiempo.

Por otro lado, la clasificación según los criterios de la AHA es limitante, ya que como se ha visto, tanto en esta serie como en otras publicadas, la frecuencia de curso atípico o incompleto es alta ${ }^{24}$, por lo que existen problemas para clasificar la enfermedad según los criterios clásicos.

La variabilidad en la presentación de la enfermedad hace necesario un mayor número de estudios de investigación que nos permitan conocer cómo se manifiesta bajo diferentes condiciones sociodemográficas y geográficas.

Se reconocen las limitaciones del estudio, pues a pesar de los esfuerzos para superar los sesgos de selección y de información hay posibilidades de afectación en la información contenida en los expedientes; además, el tamaño de la muestra es pequeño, por lo que debilita la potencia del análisis estadístico. Se sugieren estudios multicéntricos que permitan tener muestras de mayor tamaño, con análisis estadísticos potentes y la generalización de los resultados. 


\section{Responsabilidades éticas}

Protección de personas y animales. Los autores declaran que para esta investigación no se han realizado experimentos en seres humanos ni en animales.

Confidencialidad de los datos. Los autores declaran que han seguido los protocolos de su centro de trabajo sobre la publicación de datos de pacientes.

Derecho a la privacidad y consentimiento informado. Los autores han obtenido el consentimiento informado de los pacientes y/o sujetos referidos en el artículo.

\section{Conflicto de intereses}

Los autores declaran no tener ningún conflicto de intereses.

\section{Bibliografía}

1. Jamieson N, Singh-Grewal D. Kawasaki disease: a clinician's update. Int J Pediatr. 2013;2013:1-7.

2. Sotelo-Cruz N. Revisión de la enfermedad de Kawasaki en México, desde la perspectiva de las publicaciones médicas (enero de 1977 a mayo de 2012). Arch Cardiol Mex. 2013;83:214-22.

3. Gil-Veloz M, Flores-Ruiz EM, Beirana-Palenciana LG, Miranda-Novales MG, Huerta-García GC, Solórzano-Santos F. Enfermedad de Kawasaki: comportamiento clínico y complicaciones cardiovasculares en niños atendidos en un hospital de tercer nivel. Arch Cardiol Mex. 2009;79:11-7.

4. Burns JC. Commentary: translation of Dr. Tomisaku Kawasaki's original report of fifty patients in 1967. Pediatr Infect Dis J. 2002;21:993-5.

5. Kawasaki T. [Acute febrile mucocutaneous syndrome with lymphoid involvement with specific desquamation of the fingers and toes in children]. Arerugi. 1967;16:178-222.

6. Garrido-García LM, Soto-Blanquel JL, Espinosa-Rosales FJ. Enfermedad de Kawasaki: cuadro clínico, exámenes de laboratorio y lesiones coronarias. Acta Pediatr Mex. 2015;36:314-21.

7. Newburger JW, Takahashi M, Gerber MA, Gewitz MH, Tani LY, Burns JC, et al. Diagnosis, treatment, and long-term management of Kawasaki disease: a statement for health professionals from the committee on rheumatic fever, endocarditis, and Kawasaki disease, council on cardiovascular disease in the Young, American Heart Association. Circulation. 2004;110:2747-71.

8. Centers for Disease Control and Prevention. Kawasaki syndrome. USA: Department of Health \& Human Services; 2013. Disponible en: http:// www.cdc.gov/kawasaki

9. Makino N, Nakamura Y, Yashiro M, Ae R, Tsuboi S, Aoyama Y, et al. Descriptive epidemiology of Kawasaki disease in Japan, 2011-2012: from the results of the $22^{\text {nd }}$ nationwide survey. J Epidemiol. 2015;25:239-45.

10. Newburger JW, Taubert KA, Shulman ST, Rowley AH, Gewitz MH, Takahashi $\mathrm{M}$, et al. Summary and abstracts of the seventh international Kawasaki disease symposium: december 4-7, 2001, Hakone, Japan. Pediatr Res. 2003;53:153-87.

11. García-Rodríguez F, Flores-Pineda AJ, Villareal-Treviño AV, Salinas-Encinas DR, Lara-Herrera PB, Maldonado-Velásquez MR, et al. Enfermedad de Kawasaki en un hospital pediátrico en México. Bol Med Hosp Infant Mex. 2016;73:166-73.

12. Yeung RS. Kawasaki disease: update on pathogenesis. Curr Opin Rheumatol. 2012;22:551-60.

13. Rowley $\mathrm{AH}$. Kawasaki disease: novel insights into etiology and genetic susceptibility. Annu Rev Med. 2011;62:69-77.

14. Rowley AH, Shulman ST. Pathogenesis and management of Kawasaki disease. Expert Rev Anti Infect Ther. 2012;8:197-203.

15. Rowley A, Schulman S. Recent advances in the understanding and management of Kawasaki disease. Curr Infect Dis Rep. 2010;12:96-102.

16. Onouchi Y, Gunji T, Burns JC, Shimizu C, Newburger JW, Yashiro M, et al. ITPKC functional polymorphism associated with Kawasaki disease susceptibility and formation of coronary artery aneurisms. Nat Genet. 2008;40:35-42.

17. Rowley A. The complexities of the diagnosis and management of Kawasaki disease. Infect Dis Clin North Am. 2015;29:525-37.

18. Yutani C, Go S, Kamiya T, Hirose O, Misawa H, Maeda $\mathrm{H}$, et al. Cardiac biopsy of Kawasaki disease. Arch Pathol Lab Med. 1981;105:470-3.

19. Sundel RP. Kawasaki disease. Rheum Dis Clin North Am. 2015;41:63-73.

20. Morales-Quispe JA, Espínola-Zavaleta N, Caballero-Caballero R, García-López JJ, Rodríguez-Quezada JM, Betanzos-Rodríguez E. Enfermedad de Kawasaki: evolución y complicaciones cardiovasculares en niños. Rev Med Inst Mex Seguro Soc. 2011;49:295-300.

21. Sotelo N, González LA. Kawasaki disease: a rare pediatric pathology in Mexico. Twenty cases report from the Hospital Infantil del Estado de Sonora. Arch Cardiol Mex. 2007;77:299-307.

22. Surjit S, Sikha A, Sagar B, Anju G, Deepti S, Amit R, et al. Kawasaki disease in infants below 6 months: a clinical conundrum? Int J Rheum Dis. 2016;19:924-8.

23. Caballero-Mora FJ, Alonso-Martín B, Tamariz-Martel-Moreno A, Cano-Fernández J, Sánchez-Bayle M. Enfermedad de Kawasaki en 76 pacientes. Factores de riesgo de aparición de aneurismas coronarios. An Pediatr (Barc). 2011;74:232-8.

24. Sotelo-Cruz N. Curso atípico o incompleto de la enfermedad de Kawasaki en edades pediátricas. Bol Med Hosp Infant Mex. 2016;73:147-8. 